\title{
Zero sets of certain ideals of differentiable functions
}

\author{
By \\ Shuzo IzUmi \\ (Communicated by Prof. Mizohata, Aug. 20, 1975; \\ Received Feb. 19, 1976)
}

\section{Introduction}

This is an attempt to define a nice family of closed subsets on a manifold using a $C^{\infty}$-structure. Thom has proved that the zero set of a Łojasiewicz ideal is the closure of a manifold. Bochnak has obtained some results on ideals associated to closed subsets assuming that they are finitely generated (then they are Łojasiewicz ideals). But it seems that there is little known about dimensions of zero sets. If a germ $X_{a}$ of a closed subset is real analytic, its geometric dimension coincides with the algebraic one ${ }^{(\dagger)}$ (defined by the formal ideal of $X_{a}$ ). We define a family $\Phi_{a}$ of closed subsets of $\mathbf{R}^{n}$ which have similar structure to real analytic sets and whose two kinds of dimensions coincide at $a . \Phi_{a}$ is verified to be a generalization of coherent analytic sets. But its elements do not always admit a stratification.

\section{Terminology and notation}

$\mathscr{E}(U)$; the ring of $C^{\infty}$ functions on an open set $U \subset \mathbf{R}^{n}$. $\mathscr{E}_{n a}=\mathscr{E}_{a} ;$ the ring of germs of $C^{\infty}$ functions at $a \in \mathbf{R}^{n}$. in; the maximal ideal of $\mathscr{E}_{a}$.

The author wishes to express thanks to Professors: S. Nakano, M. Shiota and M. Adachi for helpful discussions and encouragement.

( $\dagger$ ) This can be verified by the last argument in [7]. 
$\mathscr{F}_{n a}=\mathscr{F}_{a}$; the ring of formal power series in the $n$ coordinate variables centered at $a$.

$\mathrm{n}$; the maximal ideal of $\mathscr{F}_{a}$.

$T_{a}: \mathscr{E}_{a} \rightarrow \mathscr{F}_{a}\left(T_{a}: \mathscr{E}(U) \rightarrow \mathscr{F}_{a}\right)$; the formal Taylor expansion at $a$.

$f \in \mathscr{E}(U)$ is flat on $A ; T_{x} f=0$ for any $x \in A$.

A closed subset $A \subset X$ is a zero of infinite order of a real valued function $f$ defined on $X \subset \mathbf{R}^{n} ; \quad\left\{x \in X:|f(x)| \leqq d(x, A)^{k}\right\}$ are neighbourhoods of $A$ in $X$ for any $k=1,2, \ldots$.

$X_{a}$; the germ of a closed subset $X \subset U$ at $a$.

$J(X, U)$ (the ideal of $X) ;\{f \in \mathscr{E}(U): f=0$ on $X\}$.

$J_{a}(X)=J\left(X_{a}\right)$ (the ideal of $X_{a}$ (of $X$ at $\left.a\right)$ ); $\left\{f \in \mathscr{E}_{a}: f=0\right.$ on $\left.X_{a}\right\}$.

$K_{a}(X)=K\left(X_{a}\right)$ (the formal ideal of $\left.X_{a}\right) ; \quad\left\{T_{a} f \in \mathscr{F}_{a}: f \in J_{a}(X)\right\}$.

$J_{a}^{\prime}(X)=J^{\prime}\left(X_{a}\right)$ (the weak ideal of $\left.X_{a}\right\} ; \quad\left\{f \in \mathscr{E}_{a}: a\right.$ is a zero of infinite order of the restriction $f \mid X\}$.

$K_{a}^{\prime}(X)=K^{\prime}\left(X_{a}\right)$ (the weak formal ideal of $\left.X_{a}\right) ; \quad\left\{T_{a} f \in \mathscr{F}_{a}: f \in J_{a}^{\prime}(X)\right\}$. $Z\left(f_{1}, f_{2}, \ldots, f_{p}\right)$; the intersection of the zero sets of $f_{1}, f_{2}, \ldots, f_{p}$.

\section{Dimensions of zero sets}

Let $X_{a}$ be the germ of a closed subset $X \subset \mathbf{R}^{n}$ at $a$. We define the geometric dimension of $X_{a}$ by $\operatorname{dim} X_{a}=\min _{a \in U: \text { open }} \operatorname{dim}(X \cap U)$, where dim denotes the inductive (=covering) dimension. The algebraic dimension $\operatorname{adim} X_{a}$ is defined by the Krull dimension of $\mathscr{F}_{a} / K_{a}(X)$, i.e. the largest number $k$ for which there exists a sequence $\left(K_{a}(X) \subset\right)$ $p_{0} \varsubsetneqq p_{1} \varsubsetneqq \cdots \sqsubseteq p_{k}=($ the maximal ideal) of prime ideals.

1. Theorem. $\operatorname{dim} X_{a}$ and $\operatorname{adim} X_{a}$ are upper semicontinuous with respect to $a$ and $\operatorname{dim} X_{a} \leqq \operatorname{adim} X_{a}$.

Proof. The semicontinuity of $\operatorname{dim}$ is obvious. As in the analytic case, there exists an affine coordinate system $(x, y)=\left(x_{1}, x_{2}, \ldots, x_{k}\right.$, $\left.y_{1}, y_{2}, \ldots, y_{l}\right)(k+l=n)$ centered at $a$ such that the canonical map $\mathscr{F}_{k}$ $\rightarrow \mathscr{F}_{n} / T_{0} \mathfrak{a}\left(\mathfrak{a}=J_{a}(X)\right)$ is injective and finite, where $\mathscr{F}_{k}$ denotes the formal power series in $x_{1}, x_{2}, \ldots, x_{k}$ (cf. [4; p. 49]). Then the map $\mathscr{E}_{k}$ $\rightarrow \mathscr{E}_{n} / \mathfrak{a}$ is also finite by Malgrange's preparation theorem. Hence $\mathscr{E}_{n} / \mathfrak{a}$ 
is integral over $\mathscr{E}_{k} / \mathscr{E}_{k} \cap \mathfrak{a}$; there exist $\sigma_{i j}(x) \in \mathscr{E}_{k}$ such that $f_{j}(x, y)$ $\equiv y_{j}^{p_{j}}+\sum_{i=0}^{p_{-j 1}} \sigma_{j i}(x) y_{j}^{i} \in \mathfrak{a}$. Then there exists a product neighbourhood $U=V \times W\left(V \subset \mathbf{R}^{k}, W \subset \mathbf{R}^{n-k}\right)$ of $a$ such that the representatives $f_{j}(x, y)$ $\equiv y_{j}^{p_{j}}+\Sigma \sigma_{j i}(x) y_{j}^{i}$ belong to $J(X, U)$. Since $\mathscr{F}_{n b} /\left(T_{b} \tilde{f}_{1}, \ldots, T_{b} \tilde{f}_{l}\right)$ is finite over $\mathscr{F}_{k b}$ its Krull dimension is less than $k$ by the theorem of Cohen and Seidenberg. Hence, $\operatorname{adim} X_{b} \leqq k$ and adim is upper semicontinuous. Let $\pi: \mathbf{R}^{n} \rightarrow \mathbf{R}^{k}$ denotes the projection $(x, y) \rightarrow(x)$. If $W$ is chosen sufficiently small the restriction $\pi \mid \pi^{-1}(U) \cap X$ is finite-to-one and proper. Then by $\operatorname{dimension}$ theory $\operatorname{dim} X \leqq \operatorname{dim} U=k$ (cf. [5; p. 63]). q.e.d.

2. Corollary. Suppose that $\operatorname{adim} X_{a}=k, K_{a}(X)$ is prime and $X$ contains $Y$ with $\operatorname{dim} Y_{a}=k$. If $f \in \mathscr{E}_{a}$ vanishes on $Y_{a}, T_{a} f \in K_{a}(X)$.

This is an analogue of the theorem of identity in the complex function theory.

To obtain the opposite estimate for $\operatorname{dim} X_{a}$ we assume that $J\left(X_{a}\right)$ is finitely generated. Let $g()$ denotes the number of the elements of a minimal basis of an ideal, which is uniquely determined for quasilocal ring (cf. [6]).

3. Proposition. Let $X \subset U$ be closed and $J_{a}(X)$ be finitely generated. Then we have:

(i) $g\left(J_{x}(X)\right)$ is upper semicontinuous with respect to $x \in U$.

(ii) $\operatorname{dim} X_{a} \geqq n-g\left(J\left(X_{a}\right)\right)$.

(iii) $\operatorname{dim}_{R} J\left(X_{a}\right) / \lll J\left(X_{a}\right)=g\left(J\left(X_{a}\right)\right)=g\left(K\left(X_{a}\right)\right)=\operatorname{dim}_{R} K\left(X_{a}\right) / ı K\left(X_{a}\right)$.

Proof. (i) Immediate from (17).

(ii) $X$ is the closure of a manifold $Y$ by (18). If $x \in Y \cap V$ ( $V$ in (17)), $J\left(X_{x}\right)$ includes the equation of $Y_{x}$. Hence

$$
\operatorname{dim} X_{a} \geqq \operatorname{dim} Y_{x} \geqq_{n} n-g\left(J\left(X_{a}\right)\right)
$$

(iii) The second equality follows from (19). The others are well known (cf. $[6 ;$ p. 13]). q.e.d.

The equality does not always hold in (ii) (cf. (12)). 


\section{The main theorem}

In this section we give a family $\Phi_{a}^{0}$ of closed sets which have a similar property to irreducible real analytic sets.

4. Definition. Let $a$ be a point of $\mathbf{R}^{n}$. $\Phi_{a}^{0}\left(\mathbf{R}^{n}\right)$ denotes the family of all closed subsets $X$ of $\mathbf{R}^{n}$ which satisfy the following:

(a) $J\left(X_{a}\right)$ is finitely generated.

(b) $K\left(X_{a}\right)$ is prime.

5. Theorem. If $X \in \Phi_{0}^{0}\left(\mathbf{R}^{n}\right)$ and if $\operatorname{adim} X_{0}=k$, then for any given neighbourhood $U_{0}$ of 0 there exist a linear subspace $\mathbf{R}^{k} \subset \mathbf{R}^{n}$, a product neighbourhood $U=V \times W \subset U_{0}\left(V \subset \mathbf{R}^{k}, W \subset \mathbf{R}^{n-k}\right)$ and $\bar{\delta} \in \mathscr{E}\left(\mathbf{R}^{n}\right)$ satisfy ing the following:

(i) $K_{0}^{\prime}(X \cap E) \cap \mathscr{F}_{k} \neq 0$ where $E=\{x \in U: \bar{\delta}(x)=0\}$.

(ii) The natural projection $\pi: X \cap U \rightarrow \mathbf{R}^{n}$ is proper. The number of points of a point inverse of $\pi$ if uniformly bounded on $V$.

(iii) $Z \equiv X \cap U \cap E^{c}$ is a nonempty $k$-dimensional manifold and $Z \mid \pi$ is a local diffeomorphism.

(iv) $\operatorname{adim}(X \cap E)_{0} \leqq k-1$.

We prove this in a more detailed form:

5'. Lemma. Let $X \in \Phi_{0}^{0}\left(\mathbf{R}^{n}\right)$ and $\operatorname{adim} X_{0}=k$. Then for any open neighbourhood $U_{0}$ of 0 there exist the following:

(1) affine coordinates $(x, y, z)=\left(x_{1}, \ldots, x_{k}, y, z_{1}, \ldots, z_{l}\right)$ centered at $0(k+1+l=n)$;

(2) a product neighbourhood $U=V \times W \subset U_{0}\left(V \subset \mathbf{R}^{k}, W \subset \mathbf{R}^{1+l}\right)$;

(3) $f(y ; x, y) \equiv y^{p}+\sum_{i=0}^{p-1} \sigma_{p-i}(x, z) y^{i} \in J(X, U)$ with discriminant $\delta(x, z)$ such that $T_{0} \sigma_{p-i} \in \mathscr{F}_{k}$ and $T_{0} f$ is prime in $\mathscr{F}_{k+1} \subset \mathscr{F}_{n}$;

(4) $g_{j}(x, y, z) \equiv \delta(x, z) z_{j}-\sum_{i=0}^{p-1} \tau_{j, p-i}(x, z) y_{i} \in J(X, U)(j=1, \ldots, l)$ such that $T_{0} \tau_{j, p-i} \in \mathscr{F}_{k}$;

(5) $\varphi(x, y, z) \in \mathscr{E}(U)$ such that $T_{0} \varphi=0$;

(6) a natural number $N$.

With that if we put 


$$
\begin{aligned}
& E=\left\{(x, y, z) \in U:\left(\delta^{N}-\varphi\right)\left(\partial\left(f, g_{1}, \ldots, g_{l}\right) / \partial(y, z)\right)=0\right\}, \\
& Y=\left\{(x, y, z) \in U: f=g_{1}=\cdots=g_{l}=0\right\}
\end{aligned}
$$

we have the following:

(7) The canonical projection $\pi: X \rightarrow \mathbf{R}^{k}$ is finite-to-one and proper;

(8) $(X \cap U) \cap E^{c}$ is a $k$-dimensional manifold with nonempty germ at 0 and the restriction $\pi \mid(X \cap U) \cap E^{c}$ is a local diffeomorphism into $\mathbf{R}^{k}$;

(9) $\operatorname{adim}((X \cap U) \cap E)_{0} \leqq k-1$;

(10) $\delta(x, z) \in J_{0}^{\prime}(X \cap E)$.

Proof. Let's put $\mathfrak{a}=J_{0}(X), \mathfrak{l}=K_{0}(X)$. By the same argument as in analytic case (cf. [4]), we can choose affine coordinates $(x, y, z)=$ $\left(x_{1}, \ldots, x_{k}, y, z_{1}, \ldots, z_{l}\right)$ of $\mathbf{R}^{n}(k+1+l=n)$ such that:

(i) The canonical map $\mathscr{F}_{k} \rightarrow \mathscr{F}_{n} / \mathscr{H}$ is injective and finite.

(ii) $\left(\mathscr{F}_{n} / \mathfrak{I I}\right)^{\sim}$ is generated by the class $\bar{y}$ of $y$ over $\widetilde{F}_{k}$. Here $\mathscr{F}_{k}$ denotes the formal power series ring in $\left(x_{1}, \ldots, x_{k}\right)$ and $\sim$ denotes the quotient field.

Let $F^{\prime}(t ; x) \equiv t^{p}+\sum_{i=0}^{p-1} S_{p-i}^{\prime}(x) t^{i}\left(S_{p-i}^{\prime}(x) \in \mathscr{F}_{k}, S_{p-i}^{\prime}(0)=0\right)$ be the minimal polynomial of $\bar{y}$ over $\widetilde{\mathscr{F}}_{k}$. Then there exists

$$
f^{\prime}(y ; x, z) \equiv y^{p}+\sum_{i=0}^{p-1} \sigma_{p-i}^{\prime}(x) y^{i}+\theta(x, y, z) \in \mathbf{a}
$$

such that $T_{0} \sigma_{p-i}^{\prime}(x)=S_{p-i}^{\prime}(x), T_{0} \theta(x, y, z)=0$. By Malgrange's preparation theorem we may assume:

(iii) $f(y ; x, z) \equiv y^{p}+\sum_{i=0}^{p-1} \sigma_{p-i}(x, z) y^{i} \in \mathfrak{a}$

such that $T_{0} \sigma_{p-i}(x, z)=S_{p-i}(x), S_{p-i}(0)=0$. Note that $z$ does not appear in $T_{0} \sigma_{p-i}(x, z)$ because of the uniqueness of the remainder in the formal preparation theorem. (There exists even a monic $f(y ; x) \in \mathfrak{a}$ with coefficients in $\mathscr{E}_{k}$ (cf. the proof of (1)). But it is not always formally minimal.) Obviously

(iii) $^{\prime} \quad F(t ; x) \equiv t^{p}+\Sigma S_{p-i}(x) t^{i} \in \mathscr{U I}$ 
is the minimal polynomial of $\bar{y}$. Let $\delta(x, z)$ and $\Delta(x)$ be the discriminants of $f(y ; x, z)$ and $F(t ; x)$ respectively. Since $\widetilde{\mathscr{F}}_{k}$ is characteristic $0, T_{0} \delta(x, z)=\Delta(x) \neq 0$. It is also the same with analytic case that:

$$
G_{j}(x, y, z) \equiv \Delta(x) z_{j}-\sum_{i=0}^{p-l} T_{j, p-i}(x) y^{i} \in \mathfrak{N l} \quad(j=1, \ldots, l)
$$

for some $T_{j, p-i}(x) \in \mathscr{F}_{k}$. Then there exist

$$
g_{j}^{\prime}(x, y, z) \equiv \delta(x, z) z_{j}-\Sigma \tau_{j, p-i}^{\prime}(x) y^{i}+\theta_{j}(x, y, z) \in \mathfrak{a}
$$

such that $T_{0} \tau_{j, p-i}^{\prime}(x)=T_{j, p-i}(x), T_{0} \theta_{j}(x, y, z)=0$. By (iii) and the preparation theorem there exist

(iv) $\quad g_{j}(x, y, z) \equiv \delta(x, z) z_{j}-\sum_{i=0}^{p-1} \tau_{j, p-i}(x, z) y^{i} \in \mathfrak{a} \quad(j=1, \ldots, l)$

with $T_{0} g_{j}(x, y, z)=G_{j}(x, y, z)$ and $T_{0} \tau_{j, p-i}(x, z)=T_{j, p-i}(x)$. As in the proof of (1) there exist

$$
h_{j}(x, z) \equiv z_{j}^{p_{j}}+\sum_{i=0}^{p_{j}-1} \sigma_{j, p-i}(x) z_{j}^{i} \in \mathfrak{a},
$$

$(\mathrm{v})^{\prime} \quad H_{j}(x, z) \equiv z_{j}^{p_{j}}+\sum_{i=0}^{p_{j}-1} S_{j, p-i}(x) z_{j}^{i} \in \mathfrak{Q I}$

with $T_{0} h_{j}(x, z)=H_{j}(x, z), T_{0} \sigma_{j, p-i}(x)=S_{j, p-i}(x)$. If we put $\mathfrak{a}=\left(e_{1}, \ldots, e_{m}\right)$ and $\mathfrak{b}=\left(f, g_{1}, \ldots, g_{l}\right)$, we have $\mathfrak{a} \supset \mathfrak{b}$. By the preparation theorem, there exist $\alpha_{\mu j}(x, y, z) \in \mathscr{E}_{n}$ and $\rho_{\mu j_{1} \ldots j_{l}}(x, y) \in \mathscr{E}_{k+1}$ such that

$$
\begin{aligned}
& e_{\mu}(x, y, z)=\sum_{j=1}^{l} \alpha_{\mu j}(x, y, z) h_{j}(x, z) \\
& +\sum_{j_{1}<p_{1}, \ldots, j_{l}<p_{l}} \rho_{\mu j_{1} \ldots j_{1}}(x, y) z_{1}^{j_{1}} \cdots z_{l}^{j_{l}} .
\end{aligned}
$$

If we put $L=\max \left\{\sum_{j=1}^{l}\left(p_{j}-1\right), p_{1}, \ldots, p_{l}\right\}$, we have

$$
\begin{gathered}
\delta^{L}(x, z) h_{j}(x, z)-\sum_{i=0}^{p-1} \rho_{j i}^{\prime}(x, z) y^{i} \in \mathfrak{b}, \\
\delta^{L}(x, z) \sum_{j_{1}, \ldots, j_{l}} \rho_{\mu j_{1} \ldots j_{l}}(x, y) z_{1}^{j_{1}} \ldots z_{l}^{j_{l}}-\sum_{i=0}^{p-1} \rho_{\mu i}^{\prime \prime}(x, z) y^{i} \in \mathfrak{b},
\end{gathered}
$$




$$
T_{0} \rho_{j i}^{\prime}(x, z) \in \mathscr{F}_{k}, \quad T_{0} \rho_{\mu i}^{\prime \prime}(x, z) \in \mathscr{F}_{k}
$$

by (iii) and (iv). Since $h_{j}$ and $\Sigma \rho_{\mu j_{1} \ldots j_{l}} z_{1}^{j_{1}} \cdots z_{l}^{j_{l}}$ are contained in $\mathfrak{a}$, $T_{0} \rho_{j i}^{\prime}=T_{0} \rho_{\mu i}^{\prime \prime}=0$ by (iii)'. Thus there exist

$$
u_{\mu}(x, y, z) \equiv \delta^{L}(x, z) e_{\mu}(x, y, z)-\rho_{\mu}(x, y, z) \in \mathfrak{b},
$$

where $\rho_{\mu}$ is flat at 0 .

Now, let $U=V \times W\left(V \subset \mathbf{R}^{k}, W \subset \mathbf{R}^{1+l}\right)$ be a product neighbourhood of 0 such that $\sigma_{p-i}, \tau_{j, p-i}, \sigma_{j, p-i}, e_{\mu}$ and $\rho_{\mu}$ have representatives in $\mathscr{E}(U)$. Hence $f(y ; x, z), \delta(x, z), g_{j}(x, z), h_{j}(x, y, z)$ and $u_{\mu}(x, y, z)$ are defined naturally as elements of $\mathscr{E}(U)$. Again we put $\mathfrak{a}=\left(e_{1}(x, y, z), \ldots\right.$, $\left.e_{m}(x, y, z)\right), \mathfrak{b}=\left(f(y ; x, z), g_{1}(x, y, z), \ldots, g_{l}(x, y, z)\right)$ as ideals of $\mathscr{E}(U)$. Narrowing $U$ if necessary, we may assume that $\mathfrak{a} \supset \mathfrak{b}, u_{\mu} \in \mathfrak{b}$ and $\mathfrak{a}$ $=J(X, U)$ by (17). We put

$$
Y=\left\{(x, y, z) \in U: f(y ; x, z)=g_{1}(x, y, z)=\cdots=g_{l}(x, y, z)=0\right\} .
$$

It is obvious that $X \cap U \subset Y$. By (20) there exist $\psi(x, y, z) \in \mathscr{E}(U)$, $\beta_{\mu}(x, y, z) \in \mathscr{E}(U)$ such that $\rho_{\mu}=\beta_{\mu}(x, y, z) \cdot \psi(x, y, z), T_{0} \psi=0$ and $\psi(x$, $y, z)>0$ if $(x, y, z) \neq(0,0,0)$. Since $\rho_{\mu}$ vanishes on $X \cap U$ by the fact $u_{\mu} \in \mathfrak{b}$, we have $\beta_{\mu} \in \mathfrak{a}$ excepting the trivial case $(0,0,0)$ is isolated in $X$. Hence there exist $\beta_{\mu v}(x, y, z) \in \mathscr{E}(U)$ such that

$$
\delta^{L} e_{\mu}-\psi \sum_{\nu=1}^{m} \beta_{\mu v} e_{v}=0 \quad(\mu=1, \ldots, m)
$$

on $Y$. Since this system of linear equations (for fixed $(x, y, z)$ ) has nontrivial solution on $Y \cap X^{c}$, we have

$$
\begin{gathered}
\operatorname{det}\left[\delta^{L}(x, z) I_{m}-\psi(x, y, z)\left(\left(\beta_{\mu v}(x, y, z)\right)\right)\right]=\delta^{L m}(x, z)-\psi(x, y, z) \beta(x, \\
y, z)=0
\end{gathered}
$$

on $Y \cap X^{c}$, where $I_{m}$ denotes the unit matrix and $\beta \in \mathscr{E}(U)$. We put

$$
\begin{aligned}
& \bar{\delta}(x, y, z)=\left(\delta^{L m}-\psi \beta\right) \cdot\left(\partial\left(f, g_{1}, \ldots, g_{l}\right) / \partial(y, z)\right), \\
& E=\{(x, y, z) \in U: \bar{\delta}=0\} .
\end{aligned}
$$

Then $Y \cap X^{c} \subset E$ and $Y \cap E^{c}$ is a $k$-dimensional manifold and $\pi: Y \cap E^{c}$ 
$\rightarrow \mathbf{R}^{k}$ is a local diffeomorphism. Since

$$
(X \cap U) \cap E^{c} \subset Y \cap E^{c} \subset Y \cap E^{c} \cap\left(Y \cap X^{c}\right)^{c}=(X \cap U) \cap E^{c},
$$

we have $Y \cap E^{c}=(X \cap U) \cap E^{c}$. It is easy to see that

$$
\begin{aligned}
& \partial\left(f, g_{1}, \ldots, g_{l}\right) / \partial(y, z) \\
& \quad=\left(\delta^{l}+\lambda\right)(\partial f / \partial y)-\sum_{j, j^{\prime}=1}^{l}\left(\delta^{l-1} \delta_{j j^{\prime}}+\lambda_{j j^{\prime}}\right)\left(\partial g_{j} / \partial y\right)\left(\partial f / \partial z_{j^{\prime}}\right),
\end{aligned}
$$

where $\lambda, \lambda_{j j^{\prime}} \in \mathscr{E}(U)$ are flat at 0 . Then, if $U$ is sufficiently small, we have

$$
|\delta|^{l+1 / 2} \leqq|\lambda| \cdot|\delta|^{1 / 2}+\sum_{j, j^{\prime}=1}^{l}\left|\delta^{l-1} \delta_{j j^{\prime}}+\lambda_{j j^{\prime}}\right| \cdot\left|\partial g_{j} / \partial y\right| \cdot M \cdot \max _{i}\left|\partial \sigma_{i} / \partial z_{j}\right|
$$

for any $(x, y, z) \in Y$ satisfying $\partial\left(f, g_{1}, \ldots, g_{l}\right) / \partial(y, z)=0$ by (22). Since $\partial \sigma_{i} / \partial z_{j}$ and $\psi \beta$ are flat at 0,0 is a zero of infinite order of the restriction $\delta \mid Y \cap E$. Since $\mathfrak{U}$ is prime and $F$ is minimal $T_{0} \delta=\Delta^{L m+l}(\partial F \mid$ $\partial t)\left.\right|_{t=y} \notin \mathfrak{R}$. This implies that $\bar{\delta} \notin \mathfrak{a}$. Then $X \cap U \cap E^{c} \neq \phi$ and $\operatorname{adim}(X$ $\cap U \cap E)_{0} \leqq k-1 . \quad y$ and $z_{1}, \ldots, z_{l}$ satisfy monic equations over $\mathscr{E}^{k}$ (such as $(v))$. Hence if $V$ is sufficiently small $\pi$ is finite to one and proper. q.e.d.

6. Corollary. In (5) we have

$$
\begin{aligned}
& \operatorname{dim}(X \cap U)=\operatorname{dim}(X \cap U)_{0}=k, \quad \operatorname{dim}(X \cap U \cap E) \leqq k-1, \\
& X \cap U \cap E \subset\{(x, y, z):|\bar{\delta}(x, y, z)| \leqq \psi(x, y, z)\}
\end{aligned}
$$

for some $\psi \in \mathscr{E}(U)$ with $T_{0} \psi=0$.

Proof. The first and the second follow from (1). The last assertion follows from (21).

\section{Reducible case and analytic case}

Let $\mathfrak{a}$ be an ideal of $E_{a}$ such that $T_{a}(\mathfrak{a})=\sqrt{T_{a}(\mathfrak{a})}$ (radical). We call it uniquely decomposable if it satisfies the following condition: 
If $T_{a}(\mathfrak{a})=\mathfrak{H}_{1} \cap \cdots \cap \mathfrak{H}_{p}$ and $\mathscr{H}_{i}=\bigcap_{j=1} P_{i j}$ such that $T_{a}(\mathfrak{a})=\bigcap_{i, j} P_{i j}$ gives the irredundant decomposition into minimal prime devisors, then there exist ideals $\mathfrak{a}_{1}, \ldots, \mathfrak{a}_{p}$ uniquely such that

$$
\mathfrak{a}=\mathfrak{a}_{1} \cap \cdots \cap \mathfrak{a}_{p}, \quad T_{a}\left(\mathfrak{a}_{i}\right)=\mathfrak{H}_{i} .
$$

7. Definition. Let $a$ be a point of $\mathbf{R}^{n} . \Phi_{a}$ denotes the family of all closed subsets $X \subset \mathbf{R}^{n}$ satisfying the following:

(a) $J\left(X_{a}\right)$ is finitely generated.

(c) $K\left(X_{a}\right)=\sqrt{K\left(X_{a}\right)}$.

(b) $J\left(X_{a}\right)$ is uniquely decomposable.

8. Proposition. If $X \in \Phi_{a}, K_{u}(X)=\mathfrak{V} \cap \mathfrak{B}, \mathfrak{N}=\bigcap_{i=1}^{p} P_{i}, \mathfrak{B}=\bigcap_{j=1}^{q} Q_{j}$ and $K_{a}(X)=\left(\cap P_{i}\right) \cap\left(\cap Q_{j}\right)$ gives the irredundant prime decomposition of $K_{a}(X)$, then the uniquely determined ideals $\mathfrak{a}, \mathfrak{b}$ with $T_{a} \mathfrak{a}=\mathfrak{H}, T_{a} \mathfrak{b}=\mathfrak{B}$ and $J_{a}(X)=\mathfrak{a} \cap \mathrm{b}$ are finitely generated. Hence their zero sets $Y_{a}$ and $Z_{a}$ are germs of elements of $\Phi_{a}$ satisfying $X_{a}=Y_{a} \cup Z_{a}$.

Proof. Let $J_{a}(X)=\left(f_{1}, \ldots, f_{r}\right) \quad$ and $\quad \mathfrak{I}=\left(T_{a} g_{1}, \ldots, T_{a} g_{s}\right), \mathfrak{B}=\left(T_{a} h_{1}, \ldots\right.$, $\left.T_{a} h_{t}\right)\left(g_{k} \in \mathfrak{a}, h_{l} \in \mathfrak{b}\right)$. Then there exist $a_{k l}, b_{k l} \in \mathscr{E}_{a}$ and $\varphi_{k} \in \mathfrak{a}, \psi_{k} \in \mathfrak{b}$ such that

$$
\begin{aligned}
& f_{k}=\sum_{l=1}^{s} a_{k l} g_{l}+\varphi_{k}=\sum_{l=1}^{1} b_{k l} h_{l}+\psi_{k}, \\
& T_{a} \varphi_{k}=T_{a} \psi_{k}=0 .
\end{aligned}
$$

Putting $\mathfrak{a}^{\prime}=\left(g_{1}, \ldots, g_{s}, \varphi_{1}, \ldots, \varphi_{r}\right), \mathfrak{b}^{\prime}=\left(h_{1}, \ldots, h_{t}, \psi_{1}, \ldots, \psi_{r}\right)$ we have $J_{a}(X)$ $=\mathfrak{a}^{\prime} \cap \mathfrak{b}^{\prime}, T_{a}\left(\mathfrak{a}^{\prime}\right)=\mathfrak{A}, T_{a}\left(\mathfrak{b}^{\prime}\right)=\mathfrak{B}$. Hence $\mathfrak{a}=\mathfrak{a}^{\prime}$ and $\mathfrak{b}=\mathfrak{b}^{\prime}$ by the uniqueness.

q.e.d.

Thus an element of $\Phi_{a}$ can be locally decomposed into a finite number of irreducible components $\left(\in \Phi_{a}^{0}\right)$ uniquely. Then by (6) we have the following:

9. Corollary. If $X \in \Phi_{a}, \operatorname{dim} X_{a}=\operatorname{adim} X_{a}$.

10. Lemma. Let $X_{1}, \ldots, X_{p} \in \Phi_{a}^{0}$ and each $X_{i}$ be the closure of 
$k_{i}$-dimensional manifold in a neighbourhood of a. Put $X=\cup_{i=1} X_{i}$ and suppose that $K_{a}(X)$ is a radical and $\left\{K_{a}\left(X_{i}\right)\right\}$ are all of its minimal prime devisors. If $J_{a}\left(\cup_{j=1}^{q} X_{i_{j}}\right)$ are finitely generated for all subfamilies $\left\{X_{i_{1}}, \ldots, X_{i_{q}}\right\} \subset\left\{X_{1}, \ldots, X_{p}\right\}, J_{a}(X)$ is uniquely decomposable and hence $X \in \Phi_{a}$.

Proof. Let $J_{a}(X)=\overbrace{j=1}^{r} \mathfrak{a}_{j}, T_{a}\left(\mathfrak{a}_{j}\right)=\overbrace{l=1}^{n_{j}} K_{a}\left(X_{j l}\right)$ such that $\left\{X_{11}, \ldots\right.$, $\left.X_{r n_{r}}\right\}=\left\{X_{1}, \ldots, X_{p}\right\}$. Suppose that $f \in \mathfrak{a}_{m} \cap J\left(\bigcup_{l=1}^{n_{m}} X_{m l}\right)^{c}$. Then $f$ does not vanish on some open subset $V$ of some $X_{m l_{0}}^{l=1}$ which is adherent to $a$. Let $k$ be the Krull dimension of $\mathscr{F}_{n} / K_{a}\left(X_{m l_{0}}\right)$. By our assumption and (5) we may assume that $V$ is a $k$-dimensional manifold. On the other hand there exists $\bar{g} \in K_{a}\left(X_{m l_{0}}\right)^{c} \cap\left(\bigcap_{j \neq m} \bigcap_{l=1}^{n_{j}} K_{a}\left(X_{j l}\right)\right)$ (cf. e.g. [6; p. 6]). Hence there exist $g_{j} \in \mathfrak{a}_{j}(j \neq m)$ such that $T_{a} g_{j}=\bar{g}$. Since $h \equiv \prod_{j \neq m} g_{j} \in$ $\bigcap_{j \neq m} \mathfrak{a}_{j}, f h \in J_{a}(X)$ and $h$ vanishes on $V$. This implies that $\bar{g} \in K_{a}\left(X_{m l_{0}}\right)$ by (2), a contradiction. Thus we have proved that $\mathfrak{a}_{m} \subset J_{a}\left(\bigcup_{l=1}^{n_{m}} X_{m l}\right)$. The converse inclusion follows from (19). These prove the uniqueness of $\mathfrak{a}_{1}, \ldots, \mathfrak{a}_{r}$.

11. Proposition. The following conditions are equivalent for a real analytic set $X$ :

(i) $X_{a}$ is coherent.

(ii) $J_{a}(X)$ is finitely generated.

(iii) $X \in \Phi_{a}$.

Proof. (i) $\Leftrightarrow$ (ii) is proved in [3]. (iii) $\Rightarrow$ (ii) is trivial. Suppose that $X_{a}$ is coherent. Let $\mathcal{O}_{n} \subset \mathscr{F}_{n}$ denote the ring of convergent power series at $a \in \mathbf{R}^{n}$ and $I_{a}(Y)$ denote the ideal of all $f \in \mathcal{O}_{n}$ vanishing on $Y$. $I_{a}(Y)$ is of course a radical. If $X=\cup_{i=1}^{p} X_{i}$ is the irreducible decomposition as an analytic germ at $a, I_{a}(X)=\bigcap_{i=1}^{p} I_{a}\left(X_{i}\right)$ is the irredundant prime decomposition. Then $\mathscr{F}_{n} \cdot I_{a}(X)=\bigcap_{i=1}^{p} \mathscr{F}_{n} \cdot I_{a}\left(X_{i}\right)$ is the irredundant prime decomposition by the theorem of Zariski and Nagata (cf. [6] or [4; p. 89]). Malgrange $\left[4 ;\right.$ p. 90] has shown that $F_{n} \cdot I_{a}(Y)=\underset{p}{K_{a}(Y)}$ for analytic $Y$. H. 
Cartan $[2 ;(13)]$ has shown that if analytic $Y=\bigcup_{i=1} Y_{i}$ is coherent any partial union $\bigcap_{j=1}^{q} Y_{i}$ is coherent, where $Y_{1}, \ldots, Y_{p}$ are irreducible components of $Y$ as an analytic set. Hence $K_{a}(X)=\bigcap_{i=1}^{p} K_{a}\left(X_{i}\right)$ is the irredundant prime decomposition and $J_{a}\left(\cup_{j=1}^{q} X_{i_{j}}\right)$ is finitely generated for any partial union $\bigcup_{j=1}^{q} X_{i j}$ of $\bigcup_{i=1}^{p} X_{i}$. This implies that $X_{1}, \ldots, X_{p} \in \Phi_{a}^{0}$. It is also known that the Krull dimension of a coherent analytic set is constant near its irreducible point. Then $X \in \Phi_{a}$ by the previous lemma. q.e.d.

\section{Reviews and examples}

Elements of $\Phi_{a}$ have still many bad properties. Especially the set of singular points of $X$ does not always belongs to $\Phi_{a}$, not even locally, and some $X$ have locally infinite topological types. Hence some $X$ admit no stratification at $a$. The author does not know whether the following hold or not:

(i) $\operatorname{dim} X_{x}$ is constant near $a$ for $X \in \Phi_{a}^{0}$.

(ii) The strong condition uniqueness in (d) of (7) is removable.

(iii) $\left\{x \in X: X \in \Phi_{x}\right\}$ is open in $X$.

(i) is affirmable if $X \in \Phi_{a}$ is analytic. As for (ii) there exists a closed set $X$ whose ideal is decomposable in infinitely many ways and whose formal ideal is a radical (cf. (15)). As for (iii) we know that the property (a) is an open property by (17). If $X$ is locally analytic (iii) holds by (11).

Now let $\varphi(x) \in \mathscr{E}(\mathbf{R})$ be zero on $(-\infty, 0]$ and positive elsewhere and $\psi(x) \in \mathscr{E}(\mathbf{R})$ be zero on $(-\infty, 0]$, flat at $1,1 / 2,1 / 3, \ldots$ and positive elsewhere.

12. Example. (Asami). Let $X=Z\left(x^{2}-y z, y^{3}-x z, z^{2}-x y^{2}\right) \subset \mathbf{R}^{3}$. Then $X$ is coherent and $J\left(X_{0}\right)=\left(x-y z, y^{3}-x z, z^{2}-x y^{2}\right)$ but $\operatorname{dim} X_{0}$ $=1>3-g\left(J\left(X_{0}\right)\right)=0$.

13. Example. Let $X=Z(y)$ and $Y=Z(y-\varphi(x))$. Then $X, Y \in \Phi_{0}\left(\mathbf{R}^{2}\right)$ but $J_{0}(X \cup Y)$ and $J_{0}(X \cap Y)$ are not finitely generated. 
14. Example. The principal ideal $(x y+\varphi(z)) \subset \mathscr{E}\left(\mathbf{R}^{3}\right)$ is not decomposable.

15. Example. Suppose that (i) $J_{a}(X)$ is finitely generated; (ii) $\mathfrak{a}$, $\mathfrak{a}_{1}, \mathfrak{a}_{2}, \ldots, \mathfrak{a}_{r}$ are ideals of $\mathscr{E}_{a}$; (iii) $\mathfrak{a} \subset J_{a}(X)$; (iv) $\stackrel{\bigcap}{i=1}_{a}^{r} T_{a} \mathfrak{a}_{i} \subset T_{a} \mathfrak{a}$; (v) $T_{a} \mathfrak{a}_{i}$ are prime; (vi) $\operatorname{dim}\left(Z\left(\mathfrak{a}_{i}\right) \cap X\right)_{a}=\operatorname{dim} \mathscr{F}_{a} / T_{a} \mathfrak{a}_{i}$. Then $J_{a}(X)=\mathfrak{a}$ by (2) and (19). From this fact the ideal $J_{a}(Y)$ of $Y=Z\left\{\left(x^{2}-z y^{2}\right)\left(y^{2}-\right.\right.$ $\left.\left.Z(x-\psi(-z))^{2}\right)\right\}$ is not finitely generated. We can easily show that $K_{a}(Y)=\sqrt{K_{a}(Y)}$ and $J_{u}(Y)$ is decomposable in infinitely many ways.

16. Example. Let's put $f=v^{2}-u\left(u+y^{2}+\psi(x)\right), \quad X=Z(f) \subset \mathbf{R}^{4}$. Since the first Jacobian extension $(f, \partial f / \partial x, \ldots, \partial f / \partial v)$ of $(f)$ is $\left(u^{2}, v\right.$, $\left.u \psi^{\prime}(x), u y, 2 u+y^{2}+\psi(x)\right)$, "the critical set" of $(f)$ is

$$
Y=\{(x, 0,0,0): x \leqq 0\} \cap\{(1,0,0,0),(1 / 2,0,0,0),(1 / 3,0,0,0), \ldots\}
$$

(cf. [1]).

(i) If $p \in X-Y, K_{p}(X)=\left(T_{p} f\right)$. If $p \in Y$ any $g \in \mathscr{E}_{4 a}$ can be expressed as $g=q f+h(x, y, u) v+k(x, y, u)\left(q \in \mathscr{E}_{4 p} ; h, k \in \mathscr{E}_{3 p}\right)$. If $u_{0}$ $\left(u_{0}+y_{0}^{2}+\psi\left(x_{0}\right)\right)>0$ there are two $v$ satisfying $\left(x_{0}, y_{0}, u_{0}, v\right) \in X$. Hence $h, k$ vanishes $u\left(u+y^{2}+\psi(x)\right)>0$ for any $g \in J_{p}(X)$. Thus $T_{p} h=T_{p} k=0$ and $K_{p}(X)=\left(T_{p} f\right)$. These mean that $J\left(X, \mathbf{R}^{4}\right)=(\bar{f})$ (the closure with respect to $C^{\infty}$-topology).

(ii) It is casy to see that $u \in \sqrt{(f, \partial f / \partial x, \ldots, \partial f / \partial v)}$ and $T_{p} u$ is not a zero divisor in $\mathscr{F}_{p} /\left(T_{p} f\right)$. Then by the theorem of Tougeron and Merrien [11], $(f)$ is closed as well as the analytic ideal $(f, u)$ $=\left(u, v^{2}\right)$.

By (i) and (ii), $J\left(X, \mathbf{R}^{4}\right)=(f)$. It is easy to see that $X \in \underset{p \in \mathbf{R}^{4}}{\cap} \Phi_{p}^{0}$. But its singular set $Y$ is locally infinite.

\section{Lemmas on funtions, ideals and sets}

Finally we list the important lemmas used in this paper.

17. Lemma. (Tougeron [9], $c f$. [3]). Let $\mathfrak{a} \subset J(X, U)$ be an ideal such that the restriction $a_{a}=J_{a}(X)$. Then there exists a neighbourhood 
$V$ of a such that the restriction $\mathfrak{a} \mid V=J(X, V)$.

18. Lemma. (Bochnak [1]). If $J(X, U)$ is finitely generated, $X$ is the closure of a manifold $Y$.

19. Lemma. ([3]). ${ }^{(\dagger)}$ Suppose that $J_{a}(X, U)$ is finitely generated. If an ideal $\mathfrak{a} \subset J_{a}(X, U)$ satisfies $T_{a} \mathfrak{a}=K_{a}(X, U)$, we have $\mathfrak{a}=J_{a}(X, U)$.

20. Lemma. (Tougeron $\left[10 ;\right.$ p. 93]). Let $f_{1}, f_{2}, \ldots \in \mathscr{E}(U)$ be flat on a closed subset $X \subset U$. Then there exists $g \in \mathscr{E}(U)$ which is strictly positive on $U-X$, flat on $X$ and $\left(f_{1}, f_{2}, \ldots\right) \subset g \cdot \underline{m}_{X}$. Where $m_{X}$ denotes the ideal of all $h \in \mathscr{E}(U)$ flat on $X$.

The proof of this lemma applies to the following:

21. Lemma. Let $f$ be a real valued function on $U$. If a closed set $X \subset U$ is a zero of infinite order of $f$, there exists $g \in \mathscr{E}(U)$ which is strictly positive on $U-X$, flat on $X$ and $|f(x)| \leqq g(x)$ on $U$.

Let $U$ be an open neighbourhood of $0 \in \mathbf{R}^{k}=\left\{\left(x_{1}, x_{2}, \ldots, x_{k}\right)\right\}$. Suppose that $\sigma_{1}(x), \sigma_{2}(x), \ldots, \sigma_{p-1}(x) \in E(U)$ and $\delta(x)$ is the discriminant of $f(y ; x)=y^{p}+\sum_{i=0}^{p-1} \sigma_{p-i}(x) y^{i}$. Then $f(y ; x)=0$ has $p$ distinct solutions $y=\varphi_{1}(x), \varphi_{2}(x), \ldots, \varphi_{p}(x)$ in $\{(x, y) \in U \times \mathbf{C}: \delta(x) \neq 0\}$.

22. Lmma. There exists a constant $M$ depending only upon $f$ and upon compact set $K \subset U$ such that

$$
\sqrt{|\delta(x)|} \cdot\left|\left(\partial \varphi_{\alpha} / \partial x_{1}\right)(x)\right| \leqq M \cdot \max _{i}\left\{\left|\left(\partial \sigma_{i} / \partial x_{1}\right)(x)\right|\right\}
$$

on $\{(x) \in K: \delta(x) \neq 0\}$,

$$
\sqrt{|\delta(x)|} \cdot\left|\left(\partial f / \partial x_{1}\right)(y ; x)\right| \leqq M \cdot \max \left|\left(\partial \sigma_{i} / \partial x_{1}\right)(x)\right| \cdot|(\partial f / \partial y)(y ; x)|
$$

on $\{(x, y) \in K \times \mathbf{C}: f(y ; x)=0\}$.

Proof. It is easy to see

( $\dagger$ A more general proposition is found in Bochnak-Risler; Quelques questions ouvertes (preprint). 


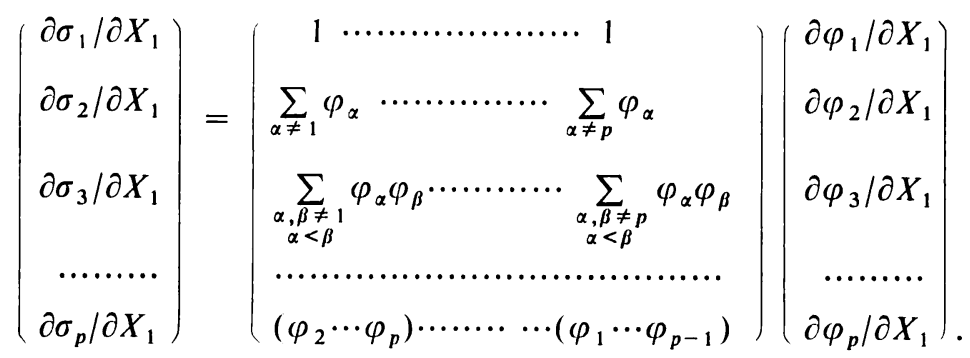

This matrix has determinant of absolute value $\sqrt{|\bar{\delta}|}$ and its elements are bounded on $\{(x) \in K: \delta(x) \neq 0\}$. Then the first inequality follows from Cramer's formula. The second holds from the fact that $\partial f / \partial x_{1}$ $=-\left(\partial \varphi_{\alpha} / \partial x_{1}\right)(\partial f / \partial y)$ on the zero set of $f$.

\section{FACUlTy OF SCIENCE AND \\ TeChNology, Kinki UNIVERSITY}

\section{References}

[1] J. Bochnak, Sur le théorèm des zéro de Hilbert "Différentiable" Topology Vol. 12 (1973) 417-424.

[2] H. Cartan, Variétés analytiques réelles et variétés analytiques complexes, Bull. Soc. Math. France, 85 (1957) 77-99.

[3] S. Izumi, Zeros of ideals of $C^{r}$ functions, J. Math. Kyoto Univ., to appear.

[4] B. Malgrange, Ideals of differentiable functions, Oxford Univ. Press (1966).

[5] J. Nagata, Modern dimension theory, Amsterdam, North-Holland (1965).

[6] M. Nagata, Local rings, New York, John Wiley \& Sons (1962).

[7] J. J. Risler, Un théorèm des zéros en géométrie analytique réelle, C. R. Acad. Sci. Paris 274, 1488-1490 (1972).

[8] R. Thom, On some ideals of differentiable functions, J. Math. Soc. Japan 19 (1967) 255-259.

[9] J. Cl. Tougeron, Faisceaux différentiables quasi-flasque. C. R. Acad. Sci. Paris 260, 2971-2973 (1965).

[10] J. Cl. Tougeron, Idéaux de fonctions différentiables, Springer (1972).

[11] J. Cl. Tougeron, J. Merrien, Idéaux de fonctions différentiables, II, Ann. Inst. Fourier 20, 179-233 (1970). 